\title{
Cosmic rays through the Higgs portal
}

\author{
Rainer Dick ${ }^{*, a, b}$, Robert B. Mann ${ }^{\mathrm{a}, \mathrm{c}}$, Kai E. Wunderle ${ }^{\mathrm{b}}$ \\ a Perimeter Institute for Theoretical Physics, 31 Caroline Street North, Waterloo, \\ Ontario, Canada N2L $2 Y 5$ \\ ${ }^{\mathrm{b}}$ Department of Physics and Engineering Physics, University of Saskatchewan, \\ 116 Science Place, Saskatoon, Saskatchewan, Canada S7N 5E2 \\ ${ }^{\mathrm{c}}$ Department of Physics and Astronomy, University of Waterloo, \\ 200 University Avenue West, Waterloo, Ontario, Canada N2L 3G1
}

\begin{abstract}
We consider electroweak singlet dark matter with a mass comparable to the Higgs mass. The singlet is assumed to couple to standard matter through a perturbative coupling to the Higgs particle. The annihilation of a singlet in the mass range $m_{S} \sim m_{h}$ is dominated by proximity to the $W, Z$ and Higgs peaks in the annihilation cross section. We find that the continuous photon spectrum from annihilation of perturbatively coupled singlets in the galactic halo can reach a level of several per mil of the EGRET diffuse $\gamma$ ray flux.
\end{abstract}

Key words: Cosmic rays, electroweak singlet, dark matter

PACS: 12.60.Fr, 95.30.Cq, 95.35.+d, 96.50.S-, 98.70.Sa

\section{Introduction}

Revealing the distribution and nature of dark matter is one of the most interesting current challenges of particle physics and astrophysics. Numerous candidates have been proposed and studied, including axions [1], neutralinos [2], Kaluza-Klein photons [3], Kaluza-Klein or string dilatons [4], and superheavy dark matter either di-

* Corresponding author

Email addresses: rainer.dick@usask.ca

(Rainer Dick),

rmann@perimeterinstitute.ca (Robert B.

Mann), kai.wunderle@usask.ca (Kai E.

Wunderle). rectly from inflationary expansion $[5,6]$ or from preheating after inflation [7]. Unstable particles like axions and dilatons have to be very light, in the sub $\mathrm{GeV}$ range, to survive long enough to serve as dark matter. However, neutralinos are usually assumed to have masses beyond $100 \mathrm{GeV}$, although lower mass limits strongly depend on supersymmetric models $[8,9,10]$.

Another very interesting model for dark matter which can have a large mass is a stable electroweak singlet $S$ which couples to standard model matter exclusively through a coupling to the Higgs boson $H$,

$H_{I}=\frac{\eta}{2} \int d^{3} \vec{x} S^{2} H^{\dagger} H$. 
We focus in particular on the $Z_{2}$ symmetric model proposed in $[11,12,13,14,15]$, which also allows for a bare mass term for the singlet, but does not include Higgs-singlet mixing terms. The Lagrangian in the scalar sector is

$$
\begin{aligned}
\mathcal{L}= & -\frac{1}{2} \partial_{\mu} S \partial^{\mu} S-\frac{1}{2} m_{S}^{2} S^{2}-D_{\mu} H^{\dagger} D^{\mu} H \\
& -\frac{\eta}{2} S^{2}\left(H^{\dagger} H-\frac{v_{h}^{2}}{2}\right) \\
& -\frac{\lambda}{4}\left(H^{\dagger} H-\frac{v_{h}^{2}}{2}\right)^{2} .
\end{aligned}
$$

The derivatives $D_{\mu}$ are the appropriate $S U(2) \times U(1)$ covariant derivatives acting on the Higgs field. We assume perturbative singlet-Higgs coupling for our calculations of singlet annihilation cross sections in the non-relativistic limit. We report results in particular for $\eta^{2}=0.1$ and for $\eta^{2}=0.01$. The singlet sector may also include a $Z_{2}$ symmetric singlet self-interaction $\sim \lambda_{S} S^{4}$ if the positive coupling $\lambda_{S}$ is weak enough such that its loop contributions can be neglected in the present perturbative calculation of singlet annihilation cross sections. The assumption of perturbative couplings in the non-relativistic limit is compatible with the fact that the $\beta$ functions are positive in leading order in the couplings [14]. We assume that the singlet vacuum expectation value vanishes, $v_{s}=\langle S\rangle=0$. Otherwise the singlet-Higgs coupling would yield a singlet-Higgs mixing term $\sim \eta v_{s} v_{h} s h$.

Later on we will also allow for a set of $N$ singlet states with a global $O(N)$ symmetry and vanishing vacuum expectation values. This ensures mass degeneracy and universality of the singlet-Higgs coupling strength $\eta$.

The Higgs-channel between dark matter and the standard model was denoted as a Higgs portal in [15]. The model provides a minimal renormalizable dark mat- ter mode $111,12,13,14,15,16,17,18]$. It has also been discussed as a model for quintessence in [22]. McDonald introduced the variant with a complex singlet [12], and effects of gauging the ensuing hidden $\mathrm{U}(1)$ symmetry are discussed in [23]. The presence of the singlet coupling obviously modifies the Higgs effective potential and impacts electroweak symmetry breaking [24], eventually triggering a strong first order phase transition $[25,26]$.

A light electroweak singlet could reveal itself as missing energy in collider based Higgs search experiments [27,28,29,23] or in B decays [17]. March-Russell et al. pointed out that electroweak singlets and their fermionic partners in supersymmetric theories can also be very heavy, with masses up to $30 \mathrm{TeV}$ [30].

In the present paper we will be concerned with the prospects of observation of intermediate mass electroweak singlets through their annihilation products in cosmic rays. We will take the proposals of an electroweak singlet coupling through the Higgs portal as a minimal renormalizable dark matter model seriously, and discuss possible annihilation signals under the assumption $m_{S} \sim m_{h}$. The relevant mechanism for a sizable signal for an intermediate mass singlet would be annihilation through an intermediate Higgs boson into $W$ and $Z$ bosons. Because of the importance of the opening of the $W$ channel at $80 \mathrm{GeV}$, we will denote the mass range $80 \mathrm{GeV}<m_{S}<1 \mathrm{TeV}$ as the intermediate mass range for electroweak singlets. Proximity of $m_{S}$ to the $W, Z$, and Higgs peaks

1 Other recently proposed classes of minimal dark matter models introduce heavy electroweak multiplets with a lightest neutral component $[19,20]$, or another scalar messenger between dark matter and standard matter [21]. 
in the annihilation cross section increases the product $v \sigma$ substantially, thus potentially yielding a strongly enhanced flux of annihilation products from the galactic halo even without invoking boost factors from strong local dark matter overdensities. However, we will see that the effect of the enhanced annihilation cross section is partially compensated for in standard Lee-Weinberg theory for the creation of thermal relics, because the requirement $\varrho_{S}=\varrho_{d m}$ together with $m_{S} \sim m_{h}$ will require $N$-plets of electroweak singlet states, and the net effect is a scaling of the flux $j \propto v \sigma / N$.

The strength of a direct galactic dark matter annihilation signal depends on the dark matter distribution in our galactic halo. The cosmic ray flux from a NavarroFrenk-White (NFW) dark matter halo and a cored isothermal halo will be reviewed in section 2. Model independent limits on the flux will be discussed in 3 .

Annihilation cross sections which follow from the singlet-Higgs coupling (1) are reported in section 4 . The application of LeeWeinberg theory for intermediate mass electroweak singlets is discussed in section 5. Section 6 summarizes our conclusions.

\section{The flux from the galactic halo}

Annihilation of dark matter particles of mass $m_{S}$ and number density $n(\vec{r})$ generates a diffuse cosmic ray flux at our location $\vec{r}_{\odot}[31]$

$$
\begin{aligned}
j= & \int d^{3} \vec{r} \frac{\nu n^{2}(\vec{r})}{4 \pi\left|\vec{r}_{\odot}-\vec{r}\right|^{2}} \\
& \times \frac{d \mathcal{N}\left(E, 2 m_{S}\right)}{d E} \frac{\sigma v}{4 \pi \mathrm{sr}} .
\end{aligned}
$$

Here $\nu=1 / 2$ if the annihilating particles are Majorana to avoid overcounting of collisions [32], and $\nu=1 / 4$ otherwise (assuming in the non-Majorana case equal amounts of dark matter and anti-matter in the halo). The case of interest to us is $\nu=1 / 2$. The fragmentation function

$\mathcal{F}\left(E, E_{\text {in }}\right)=\frac{d \mathcal{N}\left(E, E_{\text {in }}\right)}{d E}=\frac{1}{\sigma} \frac{d \sigma}{d E}$

gives the number of particles per energy interval and per annihilation event for an event with initial energy $E_{i n}$. Later on we will mostly focus on the photonic part $d \mathcal{N}_{\gamma} / d E=\sigma^{-1} d \sigma^{(\gamma)} / d E$, as charged and hadronic components of dark matter annihilation products are masked by a relatively larger cosmic ray background than photons or neutrinos. However, in the present section we will also discuss the fraction of the total flux of cosmic rays from dark matter annihilation products compared to the cosmic rays flux $j_{C R}$ from all sources.

The factor $\sigma v$ in equation (3) is the product of annihilation cross section and relative speed in the non-relativistic limit. For processes at high redshift or annihilation of very low mass particles, thermal averaging $\langle\sigma v \mathcal{F}\rangle$ would have to be included. For high redshift sources, redshifting of $d \sigma / d E$ would also have to be included and the distance $\left|\vec{r}_{\odot}-\vec{r}\right|$ has to be replaced by the luminosity distance. However, for annihilation of heavy particles in the galactic halo, equation (3) and its corresponding line-ofsight counterparts below are perfectly adequate.

Equation (3) yields a cosmic ray flux averaged over all directions. If the detector is only sensitive to cosmic rays from a small solid angle $\Delta \Omega \ll 1$ sr, or if corresponding cuts can be applied, the observed flux per unit of solid angle is [33] (see also [34]),

$j_{\Delta \Omega}=\int_{0}^{\infty} d x \int_{\Delta \Omega} d \vartheta d \varphi \sin \vartheta \frac{\nu \tilde{n}^{2}(x, \vartheta, \varphi)}{4 \pi}$ 


$$
\times \frac{d \mathcal{N}\left(E, 2 m_{S}\right)}{d E} \frac{\sigma v}{\Delta \Omega} .
$$

The vector $\vec{x}$ with length $x$ and direction $\vartheta, \varphi$ is related to the vector $\vec{r}$ in (3) through $\vec{x}=\vec{r}-\vec{r}_{\odot}, \tilde{n}(\vec{x}) \equiv n(\vec{r})$.

The averaged flux (3) is recovered from equation (4) in the following way. The observed flux for aperture $\Delta \Omega \rightarrow 0$ is the integral along the line of sight $(\vartheta, \varphi)$,

$$
\begin{aligned}
j_{\Delta \Omega \rightarrow 0}(\vartheta, \varphi)= & \int_{0}^{\infty} d x \frac{\nu \tilde{n}^{2}(x, \vartheta, \varphi)}{4 \pi} \\
& \times \frac{d \mathcal{N}\left(E, 2 m_{S}\right)}{d E} \sigma v .
\end{aligned}
$$

Averaging $j_{\Delta \Omega \rightarrow 0}(\vartheta, \varphi)$ over all directions yields the diffuse flux (3),

$$
\begin{aligned}
j= & \left\langle j_{\Delta \Omega \rightarrow 0}(\vartheta, \varphi)\right\rangle \\
= & \int_{0}^{\infty} d x \int_{0}^{\pi} d \vartheta \int_{0}^{2 \pi} d \varphi \sin \vartheta \frac{\nu \tilde{n}^{2}(x, \vartheta, \varphi)}{4 \pi} \\
& \times \frac{d \mathcal{N}\left(E, 2 m_{S}\right)}{d E} \frac{\sigma v}{4 \pi \mathrm{sr}} \\
= & \int d^{3} \vec{x} \frac{\nu \tilde{n}^{2}(\vec{x})}{4 \pi|\vec{x}|^{2}} \\
& \times \frac{d \mathcal{N}\left(E, 2 m_{S}\right)}{d E} \frac{\sigma v}{4 \pi \mathrm{sr}} .
\end{aligned}
$$

We will use equation (3) to estimate the averaged cosmic ray flux from dark matter annihilation in the galactic halo and equation (5) to estimate the diffuse cosmic ray flux along a line of sight orthogonal to the galactic plane.

We refer to the differential fluxes $(3,4)$ as inclusive fluxes. Stable final states include photons, neutrinos, electrons, positrons, protons and anti-protons. The differential photon fluxes are found by substituting $d \mathcal{N}\left(E, 2 m_{S}\right) / d E \rightarrow d \mathcal{N}_{\gamma}\left(E, 2 m_{S}\right) / d E$ in equations (3-5).
The density profile is assumed as an NFW profile [35],

$\rho(r)=\frac{M}{r\left(r+r_{s}\right)^{2}}$,

with a mass parameter $M=4.85 \times$ $10^{10} M_{\odot}=5.41 \times 10^{64} \mathrm{TeV} / c^{2}$ and a scale radius $r_{s}=21.5 \mathrm{kpc}$. These parameters correspond to the fit by Klypin et al. to the galactic halo [36], see also [37].

Equation (3) can be evaluated analytically for an NFW profile (we use $\nu=1 / 2$ in the following),

$$
\begin{aligned}
j= & \int_{0}^{\infty} d r \frac{r}{r_{\odot}} \ln \left(\frac{r+r_{\odot}}{\left|r-r_{\odot}\right|}\right) \frac{\rho^{2}(r)}{4 m_{S}^{2}} \\
& \times \frac{d \mathcal{N}\left(E, 2 m_{S}\right)}{d E} \frac{\sigma v}{4 \pi \mathrm{sr}} \\
= & \frac{M^{2}}{4 m_{S}^{2}} \frac{d \mathcal{N}\left(E, 2 m_{S}\right)}{d E} \frac{\sigma v}{4 \pi \mathrm{sr}} \frac{1}{r_{\odot} r_{s}^{4}} \\
& \times\left[\frac{\pi^{2}}{6}+\mathrm{L}_{2}\left(\frac{r_{s}}{r_{s}+r_{\odot}}\right)+\mathrm{L}_{2}\left(\frac{r_{s}-r_{\odot}}{r_{s}}\right)\right. \\
& +\frac{1}{2} \ln ^{2}\left(\frac{r_{s}+r_{\odot}}{r_{s}}\right)+\frac{4 r_{s} r_{\odot}\left(2 r_{s}^{2}-r_{\odot}^{2}\right)}{3\left(r_{s}^{2}-r_{\odot}^{2}\right)^{2}} \\
& \left.-2 r_{\odot} r_{s} \frac{9 r_{s}^{4}-8 r_{s}^{2} r_{\odot}^{2}+3 r_{\odot}^{4}}{3\left(r_{s}^{2}-r_{\odot}^{2}\right)^{3}} \ln \left(\frac{r_{s}}{r_{\odot}}\right)\right](8)
\end{aligned}
$$

where $\mathrm{L}_{2}$ denotes Euler's dilogarithmic function 2 [38,39].

Substitution of data for the galactic halo yields

$$
\begin{aligned}
& j= 1.95 \times 10^{14} \times\left(\frac{\mathrm{TeV}}{m_{S}}\right)^{2} \\
& \times \frac{d \mathcal{N}\left(E, 2 m_{S}\right)}{d E} \frac{\sigma v}{\mathrm{~cm}^{5} \mathrm{sr}} \\
&=1.95 \times 10^{14} \times\left(\frac{\mathrm{TeV}}{m_{S}}\right)^{3}
\end{aligned}
$$

$\overline{2}$ Note that $\mathrm{L}_{2}(x)=f(1-x)$ in [39]. Although of no practical relevance, we point out that the singularity at $r_{s}=r_{\odot}$ cancels between the last two terms in equation (8). 


$$
\times \frac{d \mathcal{N}\left(x, 2 m_{S}\right)}{d x} \frac{\sigma v}{\mathrm{TeV} \mathrm{cm}^{5} \mathrm{sr}},
$$

with the scaled energy variable $x=E / m_{S}$.

Looking only along a line of sight orthogonal to the galactic plane to minimize background effects will cost approximately a factor 3 in observed flux from the galactic halo,

$$
\begin{aligned}
j_{\perp}= & 6.20 \times 10^{13} \times\left(\frac{\mathrm{TeV}}{m_{S}}\right)^{3} \\
& \times \frac{d \mathcal{N}\left(x, 2 m_{S}\right)}{d x} \frac{\sigma v}{\mathrm{TeV} \mathrm{cm}^{5} \mathrm{sr}} .
\end{aligned}
$$

Cirelli et al. also compared their calculations with other density profiles [20], using the assumption of same local dark matter density. This yields for the isothermal cored profile

$\varrho=\frac{\mu}{r^{2}+r_{c}^{2}}, \quad r_{c}=5 \mathrm{kpc}$,

a parameter $\mu=6.92 \times 10^{62} \mathrm{GeV} / \mathrm{kpc}=$ $2.24 \times 10^{41} \mathrm{GeV} / \mathrm{cm}$.

The absence of the central cusp reduces the flux averaged over all directions,

$$
\begin{aligned}
j_{i s c}= & \frac{\pi \mu^{2}}{8 m_{S}^{2} r_{c}\left(r_{c}^{2}+r_{\odot}^{2}\right)} \frac{d \mathcal{N}\left(E, 2 m_{S}\right)}{d E} \frac{\sigma v}{4 \pi \mathrm{sr}} \\
= & 1.20 \times 10^{14} \times\left(\frac{\mathrm{TeV}}{m_{S}}\right)^{3} \\
& \times \frac{d \mathcal{N}\left(x, 2 m_{S}\right)}{d x} \frac{\sigma v}{\mathrm{TeV} \mathrm{cm} \mathrm{cm}^{5}}
\end{aligned}
$$

but the flux along a line of sight perpendicular to the galactic plane is virtually unchanged and slightly increased due to the weaker local gradient in the dark matter distribution,

$$
\begin{aligned}
j_{\perp}^{(i s c)}= & 6.36 \times 10^{13} \times\left(\frac{\mathrm{TeV}}{m_{S}}\right)^{3} \\
& \times \frac{d \mathcal{N}\left(x, 2 m_{S}\right)}{d x} \frac{\sigma v}{\mathrm{TeV} \mathrm{cm}^{5} \mathrm{sr}} .
\end{aligned}
$$

We will report numerical results for the flux $j$ from equation (9). The other fluxes can then easily be derived from equations (1012).

The fragmentation function into all final states must satisfy the energy sum rule

$\int_{0}^{1} d x x \frac{d \mathcal{N}(x)}{d x}=2$.

An often used parametrization is

$$
\frac{d \mathcal{N}(x)}{d x}=\frac{2 x^{\alpha}(1-x)^{\beta}}{B(\alpha+2, \beta+1)} .
$$

We will use fiducial values $\alpha=-1.5, \beta=2$ for numerical estimates. The two slope behavior of $\log (d \mathcal{N}(x) / d x)$ versus $x$ (see e.g. Fig. 17.1 in the review [40]) also suggests a phenomenological fit

$\frac{d \mathcal{N}(x)}{d x}=A \exp (-\alpha x)+B \exp (-\beta x)$

The values found by the HRS Collaboration in $e^{+} e^{-}$annihilation at $\sqrt{s}=29$ $\mathrm{GeV}$ correspond to normalized values $A=360.5, \alpha=28.4, B=97.2$ and $\beta=7.91$ [41]. A disadvantage of these 2temperature distributions is that they are very small, but do not vanish at $x=1$. However, they work very well between $0.1<x<0.9$.

The shape of fragmentation functions is only weakly energy dependent between 12 $\mathrm{GeV}$ and $202 \mathrm{GeV}$ [40], and dominated by the fragmentation properties of intermediate partons. Therefore we also use (14) for numerical work besides (13).

We will use the differential photon fragmentation function proposed in [42],

$$
\frac{d \mathcal{N}_{\gamma}(x)}{d x}=\frac{0.42 \exp (-8 x)}{x^{1.5}+0.00014},
$$

for the differential photon spectrum. This corresponds to about $11 \%$ photon energy 
yield.

\section{Model independent bounds on the cosmic ray flux from dark matter annihilation}

Although we are primarily interested in cosmic ray fluxes from the Higgs portal coupling (1), we would also like to point out that model independent estimates for cosmic rays from dark matter annihilation arise from unitarity limits and from limits on neutrino fluxes. Another model independent limit arises from halo stability [43]. However, this limit has been superseded by the neutrino limit for dark matter masses heavier than $0.1 \mathrm{GeV}[44,45]$.

The unitarity limit on s wave annihilation cross sections $\sigma \leq 4 \pi / k^{2}[46,47]$ implies

$$
\begin{aligned}
\sigma v \leq & 4.40 \times 10^{-19} \frac{\mathrm{cm}^{3}}{\mathrm{~s}} \\
& \times\left(\frac{\mathrm{TeV}}{m_{S}}\right)^{2} \frac{100 \mathrm{~km} / \mathrm{s}}{v} .
\end{aligned}
$$

Substitution in equation (9) yields a limit on the diffuse cosmic ray flux from galactic dark matter annihilation

$$
\begin{aligned}
j_{S} \leq & \frac{8.57 \times 10^{-5}}{\mathrm{TeV} \mathrm{cm} \mathrm{cm}^{2} \mathrm{sr}} \frac{d \mathcal{N}(x)}{d x} \\
& \times\left(\frac{\mathrm{TeV}}{m_{S}}\right)^{5} \frac{100 \mathrm{~km} / \mathrm{s}}{v} .
\end{aligned}
$$

Beacom et al. and Yüksel et al. recently found that limits on the diffuse cosmic neutrino signal and the halo signal can be used to impose stronger limits on the dark matter annihilation cross section for dark matter masses below $10 \mathrm{TeV}[44,45]$. Between $10 \mathrm{GeV}$ and $10 \mathrm{TeV}$, the cosmic and the isotropic halo neutrino flux limits approximately reduce the limit on the annihilation cross section $\sim m^{2}$. We take this into ac- count through a correction factor

$$
\beta_{\nu}= \begin{cases}\left(\frac{m_{S}}{10 \mathrm{TeV}}\right)^{2}, & 10 \mathrm{GeV} \leq m_{S} \leq 10 \mathrm{TeV} \\ 1, & m_{S}>10 \mathrm{TeV}\end{cases}
$$

Comparison with the cosmic ray flux below $1 \mathrm{PeV}$ [48]

$$
\begin{aligned}
j_{C R} & =\frac{2.582 \times 10^{-5}}{\mathrm{TeV} \mathrm{cm}^{2} \mathrm{~s} \mathrm{sr}}\left(\frac{\mathrm{TeV}}{E}\right)^{2.68} \\
& =\frac{1.236 \times 10^{-5}}{\mathrm{GeV} \mathrm{cm}^{2} \mathrm{~s} \mathrm{sr}}\left(\frac{100 \mathrm{GeV}}{E}\right)^{2.68}
\end{aligned}
$$

shows that a galactic dark matter annihilation signal could reach several per cent of the total differential cosmic ray flux if the annihilation cross section could get close to the upper limits either through Sommerfeld enhancement or through resonance effects. We find

$$
\begin{aligned}
\frac{j_{S}}{j_{C R}} \leq & \beta_{\nu} \times 3.32 x^{2.68} \frac{d \mathcal{N}(x)}{d x} \\
& \times\left(\frac{\mathrm{TeV}}{m_{S}}\right)^{2.32} \frac{100 \mathrm{~km} / \mathrm{s}}{v},
\end{aligned}
$$

e.g. for $m_{S}=100 \mathrm{GeV}$,

$\frac{j_{S}}{j_{C R}} \leq 6.94 \times 10^{-2} x^{2.68} \frac{d \mathcal{N}(x)}{d x} \times \frac{100 \mathrm{~km} / \mathrm{s}}{v}$.

The maximum of $x^{2.68} d \mathcal{N}(x) / d x$ for the fragmentation function (13) is 0.23 for $x=$ 0.37. For the fragmentation function (14) the maximum of $x^{2.68} d \mathcal{N}(x) / d x$ is 0.37 for $x=0.34$.

However, cosmic rays are strongly dominated by charged particles, while dark matter annihilation products are expected to contain a relatively higher neutral component of photons and neutrinos. $\gamma$ ray and neutrino observatories are therefore the primary search tools for dark matter annihilation products. We will use the diffuse photon background published by EGRET between $30 \mathrm{MeV}$ and $120 \mathrm{GeV}$ [49], 


$$
\begin{aligned}
j_{\gamma, E} & =\frac{6.89 \times 10^{-10}}{\mathrm{TeV} \mathrm{cm} \mathrm{cm} \mathrm{sr}^{2}}\left(\frac{\mathrm{TeV}}{E}\right)^{2.10} \\
& =\frac{8.68 \times 10^{-11}}{\mathrm{GeV} \mathrm{cm}^{2} \mathrm{~s} \mathrm{sr}}\left(\frac{100 \mathrm{GeV}}{E}\right)^{2.10},
\end{aligned}
$$

for benchmarking. This is the diffuse photon flux observed by EGRET after subtraction of conventional galactic sources. It has been pointed out that improved models for standard sources could reduce the EGRET signal, see [50] and references there. The sensitivity calibration beyond $1 \mathrm{GeV}$ has also been called into question [51]. The energy range between $1 \mathrm{GeV}$ and $100 \mathrm{GeV}$ is particularly relevant for continuous photon signals from dark matter in the mass range of interest here, $80 \mathrm{GeV}<m_{S}<$ $1 \mathrm{TeV}$, and we will see that signal levels may be small. Reliable further subtractions of standard sources from the diffuse $\gamma$ ray background signal, and a lower total signal, would help to identify or constrain a possible dark matter signal. EGRET sets a useful benchmark until GLAST/LAT publishes data on the diffuse $\gamma$ ray background.

Normalizing the averaged photon flux following from equation (9) to the EGRET flux (18) yields

$$
\begin{aligned}
\frac{j_{\gamma}}{j_{\gamma, E}}= & 2.83 \times 10^{23} x^{2.1} \frac{d \mathcal{N}_{\gamma}(x)}{d x} \\
& \times\left(\frac{\mathrm{TeV}}{m_{S}}\right)^{0.9} \frac{\sigma v}{\mathrm{~cm}^{3} \mathrm{~s}^{-1}} .
\end{aligned}
$$

The maximum of $x^{2.1} d \mathcal{N}_{\gamma}(x) / d x$ is 0.05 near $x \sim 0.08$. Therefore any annihilation cross section of a dark matter particle with a mass below $1.5 \mathrm{TeV}$ is constrained to

$$
\sigma v<10^{-23} \frac{\mathrm{cm}^{3}}{\mathrm{~s}} \times\left(\frac{m_{S}}{100 \mathrm{GeV}}\right)^{0.9}
$$

\section{Annihilation cross sections for the electroweak singlet}

The coupling (1) reduces in unitary gauge to

$$
H_{S h}=\frac{\eta v_{h}}{2} \int d^{3} \vec{x} S^{2} h+\frac{\eta}{4} \int d^{3} \vec{x} S^{2} h^{2} .
$$

For the annihilation of the electroweak singlet through an intermediate Higgs, we also need the couplings

$$
\begin{aligned}
& H_{h h}=\int d^{3} \vec{x} \frac{m_{h}^{2}}{2 v_{h}}\left(h^{3}+\frac{h^{4}}{4 v_{h}}\right), \\
& H_{f h}=\int d^{3} \vec{x} \sum_{f} \frac{m_{f}}{v_{h}} h \bar{f} \cdot f
\end{aligned}
$$

and

$$
\begin{aligned}
H_{W, Z h}= & \int d^{3} \vec{x}\left(2 \frac{m_{W^{2}}{ }^{2}}{v_{h}{ }^{2}} W^{-} W^{+}+\frac{m_{Z}{ }^{2}}{v_{h}{ }^{2}} Z^{2}\right) \\
& \times\left(v_{h} h+\frac{h^{2}}{2}\right) .
\end{aligned}
$$

These couplings yield the following annihilation cross sections in the nonrelativistic limit,

$$
\begin{aligned}
v \sigma_{S S \rightarrow h h}= & \eta^{2} \frac{\sqrt{m_{S}{ }^{2}-m_{h}{ }^{2}}}{16 \pi m_{S}{ }^{3}} \\
& \times \mid \frac{2 m_{S}{ }^{2}+m_{h}{ }^{2}}{4 m_{S}^{2}-m_{h}{ }^{2}+\mathrm{i} m_{h} \Gamma_{h}} \\
& -\left.\frac{2 \eta v_{h}{ }^{2}}{2 m_{S}{ }^{2}-m_{h}^{2}}\right|^{2}
\end{aligned}
$$

$$
\begin{aligned}
v \sigma_{S S \rightarrow f \bar{f}}= & \eta^{2} \frac{N_{c} m_{f}^{2}}{4 \pi m_{S}^{3}} \\
& \times \frac{{\sqrt{m_{S}^{2}-m_{f}^{2}}}^{3}}{\left(4 m_{S}^{2}-m_{h}^{2}\right)^{2}+m_{h}^{2} \Gamma_{h}^{2}}, \\
v \sigma_{S S \rightarrow W W}= & \eta^{2} \frac{{\sqrt{m_{S}^{2}-m_{W}^{2}}}_{4 \pi m_{S}^{3}}}{}
\end{aligned}
$$




$$
\begin{aligned}
& \times \frac{3 m_{W}{ }^{4}-4 m_{W}{ }^{2} m_{S}{ }^{2}+4 m_{S}{ }^{4}}{\left(4 m_{S}{ }^{2}-m_{h}{ }^{2}\right)^{2}+m_{h}{ }^{2} \Gamma_{h}{ }^{2}}, \\
v \sigma_{S S \rightarrow Z Z}= & \eta^{2} \frac{\sqrt{m_{S}^{2}-m_{Z}^{2}}}{8 \pi m_{S}^{3}} \\
& \times \frac{3 m_{Z}^{4}-4 m_{Z}{ }^{2} m_{S}{ }^{2}+4 m_{S}{ }^{4}}{\left(4 m_{S}{ }^{2}-m_{h}\right)^{2}+m_{h}{ }^{2} \Gamma_{h}{ }^{2}},
\end{aligned}
$$

with $N_{c}=3$ for quarks and $N_{c}=1$ for leptons. The $\sigma_{S S \rightarrow f \bar{f}}$ cross section is summed over final spin states. The cross section $\sigma_{S S \rightarrow h h}$ contains the scattering amplitude from the $S^{2} h^{2}$ contact vertex in equation (20), the scattering amplitude from the $s$-channel contribution with an intermediate Higgs boson from the $S^{2} h$ vertex in equation (20) and the $h^{3}$ vertex in equation (21), and the $t$-channel and $u$-channel amplitudes with an intermediate singlet from the $S^{2} h$ vertex. Those amplitudes are with the normalization $S_{f i}=\delta_{f i}-\mathrm{i} \mathcal{M}_{f i} \delta^{4}\left(p_{1}+p_{2}-k_{1}-k_{2}\right)$,

$$
\begin{aligned}
\mathcal{M}_{S S \rightarrow h h}^{(1)}= & \frac{\eta}{16 \pi^{2}} \\
& \times \frac{1}{\sqrt{E_{S}\left(\vec{k}_{1}\right) E_{S}\left(\vec{k}_{2}\right) E_{h}\left(\vec{p}_{1}\right) E_{h}\left(\vec{p}_{2}\right)}}, \\
\mathcal{M}_{S S \rightarrow h h}^{(2)}= & -\frac{3 \eta m_{h}^{2}}{16 \pi^{2}} \\
& \times \frac{1}{\sqrt{E_{S}\left(\vec{k}_{1}\right) E_{S}\left(\vec{k}_{2}\right) E_{h}\left(\vec{p}_{1}\right) E_{h}\left(\vec{p}_{2}\right)}} \\
& \times \frac{1}{\left(k_{1}+k_{2}\right)^{2}+m_{h}^{2}-\mathrm{i} \epsilon}, \\
\mathcal{M}_{S S \rightarrow h h}^{(3)}= & -\frac{\eta^{2} v_{h}^{2}}{8 \pi^{2}} \\
& \times \frac{1}{\sqrt{E_{S}\left(\vec{k}_{1}\right) E_{S}\left(\vec{k}_{2}\right) E_{h}\left(\vec{p}_{1}\right) E_{h}\left(\vec{p}_{2}\right)}} \\
& \times\left(\frac{1}{\left(k_{1}-p_{1}\right)^{2}+m_{S}^{2}-\mathrm{i} \epsilon}\right. \\
& \left.+\frac{1}{\left(k_{1}-p_{2}\right)^{2}+m_{S}^{2}-\mathrm{i} \epsilon}\right) .
\end{aligned}
$$

Note that due to the constraint $m_{S}{ }^{2}>$ $m_{h}{ }^{2}$ for $S S \rightarrow h h$, neither the $s$-channel resonance for $m_{S}=m_{h} / 2$ nor the $t$-channel or $u$-channel resonance for $m_{S}=m_{h} / \sqrt{2}$ can be realized for $\sigma_{S S \rightarrow h h}$. Furthermore, $\Gamma_{S}=0$ in our models.

The amplitude for annihilation of two singlets with momenta $\vec{k}_{1}$ and $\vec{k}_{2}$ into massive vector bosons with momenta $\vec{p}_{1}, \vec{p}_{2}$ and polarizations $\epsilon^{(\alpha)}\left(\vec{p}_{1}\right), \epsilon^{(\beta)}\left(\vec{p}_{2}\right)$ is proportional to $m_{W}{ }^{2}$,

$$
\begin{aligned}
& \mathcal{M}_{S S \rightarrow W W}=-\frac{\eta m_{W}{ }^{2}}{8 \pi^{2}} \\
& \times \frac{1}{\sqrt{E_{S}\left(\vec{k}_{1}\right) E_{S}\left(\vec{k}_{2}\right) E_{W}\left(\vec{p}_{1}\right) E_{W}\left(\vec{p}_{2}\right)}} \\
& \times \frac{\epsilon^{(\alpha)}\left(\vec{p}_{1}\right) \cdot \epsilon^{(\beta)}\left(\vec{p}_{2}\right)}{\left(k_{1}+k_{2}\right)^{2}+m_{h}^{2}-\mathrm{i} \varepsilon}
\end{aligned}
$$

However, the longitudinal polarization vector for massive vector bosons comes with a factor $m_{W}^{-1}$, e.g. the polarization vectors for $\vec{p}$ in $z$-direction are

$$
\begin{aligned}
& \epsilon^{(1)}(\vec{p})=(0,1,0,0), \quad \epsilon^{(2)}(\vec{p})=(0,0,1,0), \\
& \epsilon^{(3)}(\vec{p})=\frac{1}{m_{W}}\left(|\vec{p}|, 0,0, \sqrt{\vec{p}^{2}+m_{W^{2}}}\right) .
\end{aligned}
$$

The tensor product of massive polarization vectors

$\sum_{\alpha} \epsilon^{(\alpha) \mu}(\vec{p}) \otimes \epsilon^{(\alpha) \nu}(\vec{p})=\eta^{\mu \nu}+\frac{p^{\mu} p^{\nu}}{m_{W}{ }^{2}}$

implies

$$
\begin{aligned}
& \sum_{\alpha, \beta}\left(\epsilon^{(\alpha)}\left(\vec{p}_{1}\right) \cdot \epsilon^{(\beta)}\left(\vec{p}_{2}\right)\right)^{2} \\
& =\left(\eta^{\mu \nu}+\frac{p_{1}^{\mu} p_{1}^{\nu}}{m_{W}^{2}}\right)\left(\eta_{\nu \mu}+\frac{p_{2 \nu} p_{2 \mu}}{m_{W}{ }^{2}}\right) \\
& =2+\frac{\left(p_{1} \cdot p_{2}\right)^{2}}{m_{W}{ }^{4}} .
\end{aligned}
$$

Together with the amplitude (24) this yields the cross section $\sigma_{S S \rightarrow W W}$. An equivalent way to understand why the cross 
sections $\sigma_{S S \rightarrow W W}$ and $\sigma_{S S \rightarrow Z Z}$ do not vanish in the limit of vanishing coupling $v_{h}{ }^{2} \sim m_{W}{ }^{2} \sim m_{Z}{ }^{2} \rightarrow 0$ are the residual Goldstone boson modes $h^{ \pm}$and $\chi^{0}$ from the Higgs field, which would contribute to singlet annihilation in the $S U(2) \times U(1)$ symmetric limit.

Standard model decay widths of the Higgs particle are small unless the Higgs boson is heavy enough to decay into weak gauge bosons [52], e.g. $\Gamma_{h}\left(m_{h}=\right.$ $115 \mathrm{GeV})=4.56 \mathrm{MeV}, \Gamma_{h}\left(m_{h}=160 \mathrm{GeV}\right)=$ $6.37 \mathrm{MeV}, \Gamma_{h}\left(m_{h}=165 \mathrm{GeV}\right)=247 \mathrm{MeV}$, $\Gamma_{h}\left(m_{h}=203 \mathrm{GeV}\right)=1.54 \mathrm{GeV}$.

The energy dependent cross sections $\sigma v(K)$ in order $\mathcal{O}\left(\eta^{2}\right)$ in all cases correspond to the substitution

$m_{S}^{2} \rightarrow\left(K+m_{S}\right)^{2}$

in the equations for $v \sigma$, where $K$ is the kinetic energy of an electroweak singlet. The non-relativistic limits for $v \sigma$ are therefore not only excellent approximations for $T \ll m_{S}$, but also provide upper limits on the thermal averages at high temperature

$$
\langle\sigma v\rangle=\frac{\int_{0}^{\infty} d K \frac{\left(K+m_{S}\right) \sqrt{K\left(K+2 m_{S}\right)}}{\exp \left[\left(K+m_{S}\right) / T\right]-1} \sigma v(K)}{\int_{0}^{\infty} d K \frac{\left(K+m_{S}\right) \sqrt{K\left(K+2 m_{S}\right)}}{\exp \left[\left(K+m_{S}\right) / T\right]-1}} .
$$

The temperature dependence is very weak. The $\mathcal{O}\left(\eta^{2}\right)$ cross section for $m_{S}=$ $200 \mathrm{GeV}$ is only reduced from $\sigma v=$ $1.04 \eta^{2} \times 10^{-23} \mathrm{~cm}^{3} / \mathrm{s}$ at $T=0$ to $\langle\sigma v\rangle=$ $0.39 \eta^{2} \times 10^{-23} \mathrm{~cm}^{3} / \mathrm{s}$ at $T=100 \mathrm{GeV}$.

The tree-level cross sections reported here yield cross sections of order $\sigma v \sim$ $\eta^{2} \times 10^{-23} \mathrm{~cm}^{3} / \mathrm{s}$ in the mass range $m_{S} \sim m_{h}$, see also figures 1-5 below. In addition there are also loop suppressed photon lines at $E_{\gamma}=m_{S}$ from a $\gamma \gamma$ final state, and at $E_{\gamma}=m_{S}-\left(m_{Z}^{2} / 4 m_{S}\right)$ from a $\gamma Z$ state. These contributions are of order of a per cent compared to the continuous spectrum, because the loop amplitudes contain two vertices of electroweak strength or the direct $W^{+} W^{-} \rightarrow \gamma \gamma$ contact vertex. The contribution to $v \sigma_{S S \rightarrow \gamma \gamma}$ through a $W^{+} W^{-}$ loop with contact vertex e.g. is approximately of order (neglecting logarithmic mass dependencies)

$$
\begin{aligned}
v \sigma_{S S \rightarrow \gamma \gamma} & \sim \frac{32 \pi \alpha^{2} \eta^{2} m_{W}{ }^{4}}{m_{S}^{2}\left[\left(4 m_{S}^{2}-m_{h}^{2}\right)^{2}+m_{h}{ }^{2} \Gamma_{h}{ }^{2}\right]} \\
& \sim 10^{-3} v \sigma_{S S} .
\end{aligned}
$$

The continuous spectrum yields a conservatively estimated photon energy yield of order $10 \%$ (see equation (15)). Together with the ratio of cross sections, this implies that in terms of photon energy yields from singlet annihilation, the line spectrum should be of order of a few per cent of the continuous spectrum. Therefore we focus on the continuous spectrum in the present investigation. However, further study of the loop induced line spectrum is also of interest.

\subsection{Annihilation signal from a heavy elec- troweak singlet in tree level approxi- mation}

If we assume a Higgs mass limit $m_{h} \leq$ $203 \mathrm{GeV}$ from electroweak analysis [53] and absence of a very heavy fourth generation below $m_{S}$, we get in leading order the following annihilation cross section for a heavy $\left(m_{S}>1 \mathrm{TeV}\right)$ electroweak singlet coupling through the Higgs portal,

$$
\begin{aligned}
v \sigma_{S S} & \simeq \frac{7 \eta^{2}}{64 \pi m_{S}^{2}} \\
& =4.06 \eta^{2} \times 10^{-25}\left(\frac{\mathrm{TeV}}{m_{S}}\right)^{2} \frac{\mathrm{cm}^{3}}{\mathrm{~s}} .
\end{aligned}
$$

This translates into a cosmic ray flux from heavy electroweak singlet annihila- 
tion through the Higgs portal at tree level,

$j_{S} \simeq \frac{7.92 \eta^{2} \times 10^{-11}}{\mathrm{TeV} \mathrm{cm}^{2} \mathrm{~s} \mathrm{sr}}\left(\frac{\mathrm{TeV}}{m_{S}}\right)^{5} \frac{d \mathcal{N}(x)}{d x}$

This is very small compared to the galactic background flux from supernovae and their remnants, and again photon signals are expected to be more sensitive. Normalizing to the EGRET flux formally yields

$\frac{j_{\gamma}}{j_{\gamma, E}}=0.115 \eta^{2} x^{2.1} \frac{d \mathcal{N}_{\gamma}(x)}{d x}\left(\frac{\mathrm{TeV}}{m_{S}}\right)^{2.9}$.

Due to the energy limit of EGRET this should only be used for $x<0.12 \mathrm{TeV} / m_{S}$. For $m_{S}=1 \mathrm{TeV}$ and $E \simeq 76 \mathrm{GeV}$ we find $j_{\gamma} / j_{\gamma, E} \simeq 5.6 \eta^{2} \times 10^{-3}$.

For heavier singlets, March-Russell et al. have pointed out that Sommerfeld enhancement due to scalar exchange between annihilating supersymmetric singlets can boost annihilation cross sections by factors $10^{3}$ to $10^{5}$ for singlet masses between $1 \mathrm{TeV}$ and $30 \mathrm{TeV}$ [30]. Sommerfeld enhanced annihilation signals from heavy electroweak multiplets have recently been discussed by Cirelli et al. [20]. In the present paper, we will instead focus on electroweak singlet annihilation in the intermediate mass range $80 \mathrm{GeV}<m_{S}<1 \mathrm{TeV}$, when $\sigma_{S S}$ is enhanced due to proximity to the $W, Z$ and Higgs peaks in the cross section.

\subsection{Enhancement of electroweak singlet annihilation for $m_{S} \sim m_{h}$ due to the $W, Z$ and Higgs peaks}

Electroweak singlet annihilation through an intermediate Higgs can be strongly enhanced when the channels $S S \rightarrow W W$ and $S S \rightarrow Z Z$ open up. This is especially relevant when $m_{S} \sim m_{h}$, which is the primary mass range of interest in our present investigation.
We will primarily use Higgs mass values $m_{h}=115 \mathrm{GeV}$ from the direct search limit [53], and $m_{h}=160 \mathrm{GeV}$ in the range of highest sensitivity for search for a light Standard Model Higgs boson at the Tevatron [54]. The value $m_{h}=160 \mathrm{GeV}$ is also in the preferred mass range for minimal dark matter models identified in reference [14].

Compared to $\sigma_{S S \rightarrow W W}$ the contributions of light quarks and leptons to $\sigma_{S S}$ are of order $10^{-4}$ and the contributions of heavy quarks and leptons are of order $10^{-2}$ in the mass range of interest. We will include $\mathrm{c}, \mathrm{b}$ and t quarks with masses $m_{c}=1.25 \mathrm{GeV}$, $m_{b}=4.20 \mathrm{GeV}$ and $m_{t}=172.5 \mathrm{GeV}$ in the calculation of $v \sigma_{S S}$. The $\tau$ lepton will be included with $m_{\tau}=1.78 \mathrm{GeV}$.

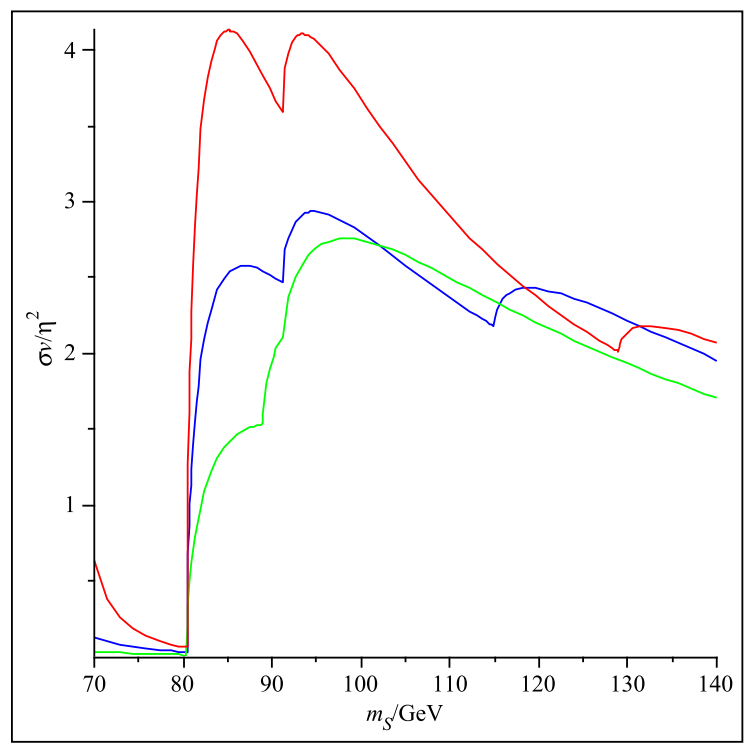

Fig. 1. The cross section $\eta^{-2} v \sigma_{S S}$ in units of $10^{-23} \mathrm{~cm}^{3} / \mathrm{s}$ for very weak coupling $\eta^{2} \lesssim 0.01$. The red (initially and finally highest) curve is for $m_{h}=129 \mathrm{GeV}$, the blue (initially and finally middle) curve is for $m_{h}=115 \mathrm{GeV}$, and the green (initially and finally lowest) curve is for $m_{h}=89 \mathrm{GeV}$.

The effect of the $t$-channel plus $u$-channel amplitude $\mathcal{M}_{S S \rightarrow h h}^{(3)}$ is small for very weak coupling $\eta^{2} \lesssim 0.01$. The $\mathcal{O}\left(\eta^{2}\right)$ cross sections for very weak coupling are displayed 
for several Higgs mass values in figures 1 and 2 .

Figure 1 shows the scaled cross section $\eta^{-2} v \sigma_{S S}$ for $m_{h}=115 \mathrm{GeV}$ and $70 \mathrm{GeV}<m_{S}<140 \mathrm{GeV}$. The cross sections for $m_{h}=89 \mathrm{GeV}$ and $m_{h}=129 \mathrm{GeV}$ are included for comparison. The edge at $80 \mathrm{GeV}$ arises from the opening of the $W$ channel $S S \rightarrow W W$, the edge at $91 \mathrm{GeV}$ arises from the $Z$ channel, and the edge at $m_{S}=m_{h}$ arises from the Higgs channel $S S \rightarrow h h$.



Fig. 2. The cross section $\eta^{-2} v \sigma_{S S}$ in units of $10^{-23} \mathrm{~cm}^{3} / \mathrm{s}$ for very weak coupling $\eta^{2} \lesssim 0.01$. The blue (initially and finally lower) curve is the cross section for $m_{h}=160 \mathrm{GeV}$. The red (initially and finally upper) curve is the cross section for $m_{h}=165 \mathrm{GeV}$.

The scaled cross sections $\eta^{-2} v \sigma_{S S}$ for $m_{h}=160 \mathrm{GeV}$ and $m_{h}=165 \mathrm{GeV}$ are displayed in figure 2.

The cross sections for larger values of $m_{S}$ approach the asymptotic limit (25), see figure 3.

For electroweak strength coupling $\eta^{2}=$ 0.1 , the $t$-channel plus $u$-channel amplitude $\mathcal{M}_{S S \rightarrow h h}^{(3)}$ suppresses the Higgs threshold in the cross sections, see figures 4 and 5 .

The continuous photon spectrum for

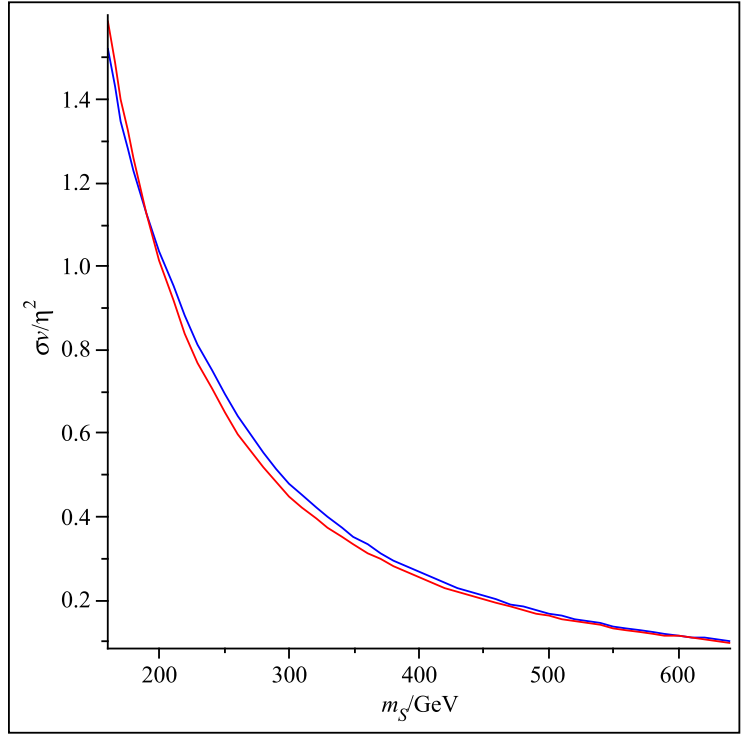

Fig. 3. The cross section $\eta^{-2} v \sigma_{S S}$ in units of $10^{-23} \mathrm{~cm}^{3} / \mathrm{s}$ in the mass range $160 \mathrm{GeV}<m_{S}<640 \mathrm{GeV}$ for very weak coupling $\eta^{2} \lesssim 0.01$. The red (initially upper) curve is the asymptotic cross section (25). The blue (initially lower) curve is for $m_{h}=115 \mathrm{GeV}$. The cross section for $m_{h}=160 \mathrm{GeV}$ also differs by less than 10\% from the aymptotic limit for $m_{S}>400 \mathrm{GeV}$.

$E_{\gamma}<120 \mathrm{GeV}$ in units of the EGRET flux is displayed in figure 6 for $m_{S}=120$ $\mathrm{GeV}, m_{h}=115 \mathrm{GeV}, \eta^{2}=0.1$ and $v \sigma_{S S}=2.03 \times 10^{-24} \mathrm{~cm}^{3} / \mathrm{s}$.

The corresponding flux ratio for $m_{S}=$ $120 \mathrm{GeV}, m_{h}=160 \mathrm{GeV}, \eta^{2}=0.1$ and $v \sigma_{S S}=3.87 \times 10^{-24} \mathrm{~cm}^{3} / \mathrm{s}$ is diplayed in figure 7 .

The fluxes in figures 6 and 7 assume that all cold dark matter is in a singlet state, but do not take into account correlations between $m_{S}$ and $m_{h}$ from thermal creation of singlets. This topic will be addressed in the next section.

\section{Thermal creation and abundance estimates}

A thorough and beautiful application of Lee-Weinberg theory [55] for abundance 


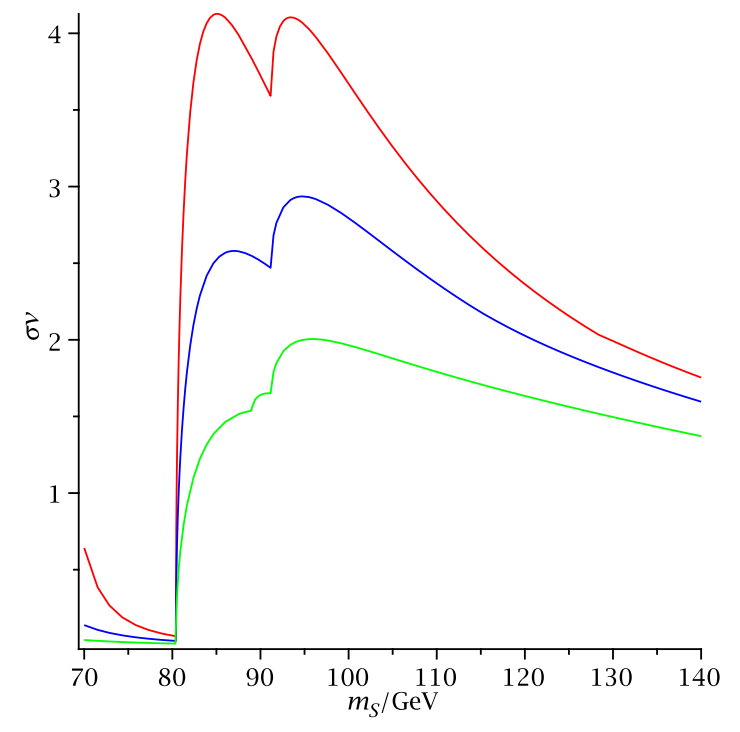

Fig. 4. The cross section $v \sigma_{S S}$ in units of $10^{-24} \mathrm{~cm}^{3} / \mathrm{s}$ for coupling $\eta^{2}=0.1$. The red (initially and finally highest) curve is for $m_{h}=129 \mathrm{GeV}$, the blue (initially and finally middle) curve is for $m_{h}=115 \mathrm{GeV}$, and the green (initially and finally lowest) curve is for $m_{h}=89 \mathrm{GeV}$.

estimates of electroweak singlets has been given in [12]. We will revisit the subject for the particular range of masses and cross sections of interest to us. The application of Lee-Weinberg theory in reference [12] used $\Omega_{d m}=1$ while the application for light electroweak singlets in [13] used $\Omega_{d m}=0.6$. Here we use $\Omega_{d m}=0.2$ [53].

In the absence of more detailed model assumptions about singlet generating interactions behind the Higgs portal or coannihilations, the effect of thermal creation of electroweak singlets below the electroweak phase transition is taken into account through a thermal production term in the rate equation

$\frac{d}{d t}\left(n a^{3}\right)=\dot{N}_{\text {thermal }}-\langle\sigma v\rangle n^{2} a^{3}$,

where $a(t)$ is the scale factor in the Robertson-Walker metric. The thermal production term is determined from the

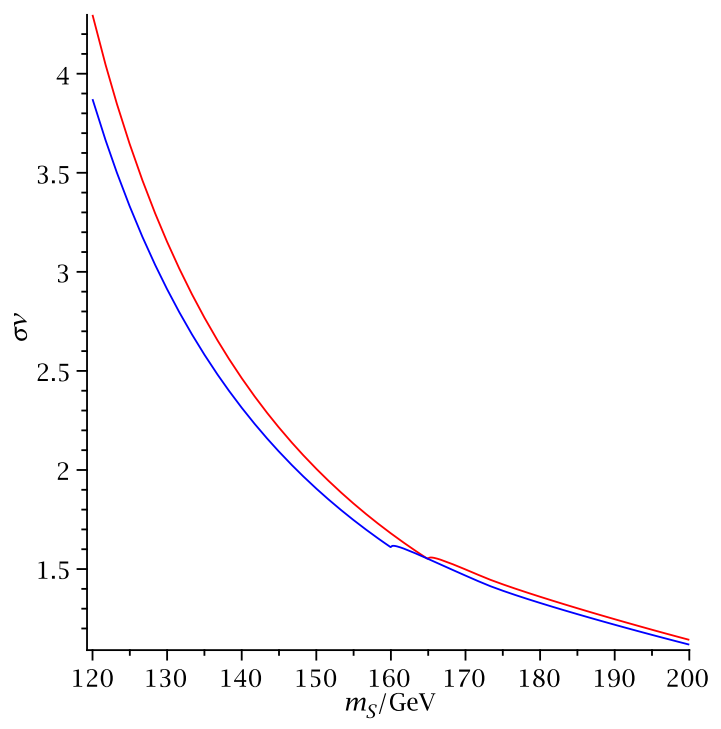

Fig. 5. The cross section $v \sigma_{S S}$ in units of $10^{-24} \mathrm{~cm}^{3} / \mathrm{s}$ for coupling $\eta^{2}=0.1$. The blue (initially and finally lower) curve is the cross section for $m_{h}=160 \mathrm{GeV}$. The red (initially and finally upper) curve is the cross section for $m_{h}=165 \mathrm{GeV}$.

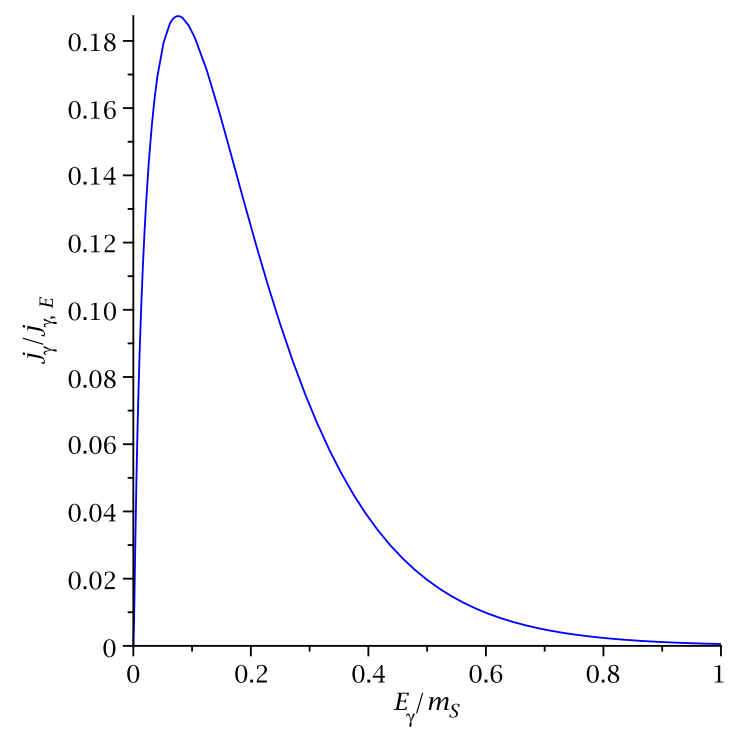

Fig. 6. The photon flux $j_{\gamma}$ in the continuous spectrum for $m_{h}=115 \mathrm{GeV}, m_{S}=120 \mathrm{GeV}$, $\eta^{2}=0.1$ and $E_{\gamma}<120 \mathrm{GeV}$ in units of the EGRET flux.

equilibrium requirement $d\left(n a^{3}\right) / d t=0$ $[55,56]$,

$\dot{N}_{\text {thermal }}=\langle\sigma v\rangle n_{0}^{2} a^{3}$, 


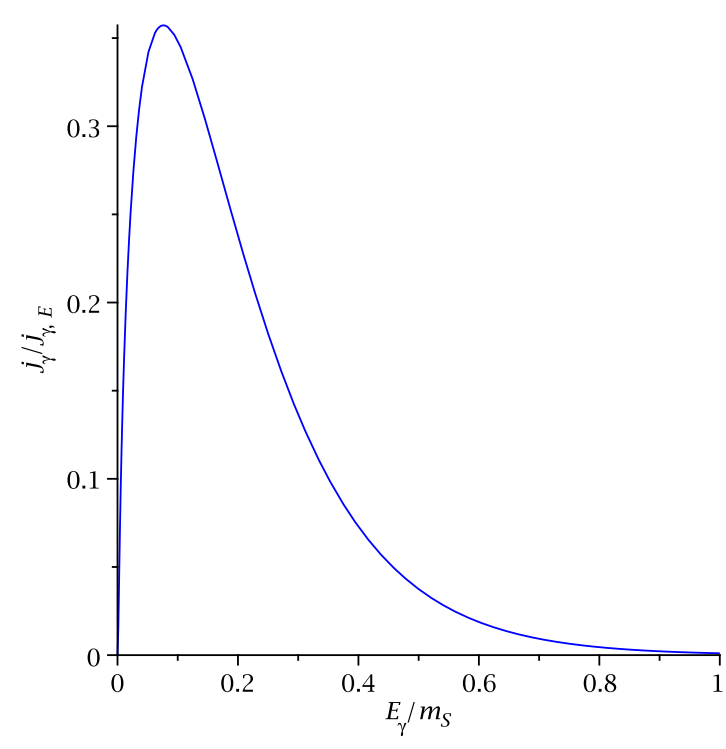

Fig. 7. The photon flux $j_{\gamma}$ in the continuous spectrum for $m_{h}=160 \mathrm{GeV}, m_{S}=120 \mathrm{GeV}$, $\eta^{2}=0.1$ and $E_{\gamma}<120 \mathrm{GeV}$ in units of the EGRET flux.

where $n_{0}$ is the thermal equilibrium density for temperature $T$. The resulting rate equations during radiation domination is

$\frac{d n}{d t}+\frac{3 n}{2 t}=-\langle\sigma v\rangle\left(n^{2}-n_{0}^{2}\right)$.

Radiation domination also yields

$t=\frac{b}{T^{2}}$,

and this is used to rewrite equation (28) in the form

$\frac{d}{d T} \frac{n}{T^{3}}=2 b\langle\sigma v\rangle \frac{n^{2}-n_{0}^{2}}{T^{6}}$.

Equation (29) follows from the relations for the energy density in radiation for $t_{\text {inflation }} \ll t<t_{\text {eq }}\left(t_{e q} \simeq 2.4 \times 10^{12} \mathrm{~s}\right.$ is the time of radiation-matter equality),

$\varrho_{\gamma}=g_{*}(T) \frac{\pi^{2}\left(k_{B} T\right)^{4}}{30(\hbar c)^{3}}=\frac{3 m_{\text {Planck }}^{2} c}{4 \hbar t^{2}}$.

Here we use the reduced Planck mass $m_{\text {Planck }}=\left(\hbar c / 8 \pi G_{N}\right)^{1 / 2}$. We have $g_{*}(T)=$ 91.5 for $m_{b}<T<m_{W}$. The parameter $b$ is

$$
\begin{aligned}
b & =\frac{3 \hbar m_{\text {Planck }} c^{2}}{\pi k_{B}^{2}} \sqrt{\frac{5}{2 g_{*}(T)}} \\
& =2.53 \times 10^{-7} k_{B}^{-2} \mathrm{~s} \mathrm{GeV}^{2} \\
& =3.41 \times 10^{19} \mathrm{~s} \mathrm{~K}^{2} .
\end{aligned}
$$

The analytic approximation proposed by Lee and Weinberg [55] uses the equilibrium density

$$
\begin{aligned}
& n_{0}(T)=\frac{1}{2 \pi^{2}(\hbar c)^{3}} \\
& \times \int_{0}^{\infty} d K \frac{\left(K+m c^{2}\right) \sqrt{K\left(K+2 m c^{2}\right)}}{\exp \left[\left(K+m c^{2}\right) / k_{B} T\right]-1}
\end{aligned}
$$

until a freeze-out temperature $T_{f}$ is reached with

$$
\left.\frac{d}{d T} \frac{n_{0}(T)}{T^{3}}\right|_{T=T_{f}}=2 b\langle\sigma v\rangle \frac{n_{0}^{2}\left(T_{f}\right)}{T_{f}^{6}} .
$$

This temperature usually turns out to satisfy $T_{f} \lesssim 0.05 m_{S}$, such that the nonrelativistic limit of $n_{0}(T)$,

$$
n_{0}(T)=\left(\frac{1}{\hbar} \sqrt{\frac{m_{S} k_{B} T}{2 \pi}}\right)^{3} \exp \left(-\frac{m_{S} c^{2}}{k_{B} T}\right),
$$

can be used in the evaluation of the LeeWeinberg condition (32). The solution is then extended for $T<T_{f}$ using domination of the annihilation term in the rate equation (26),

$\frac{d n}{d t}+\frac{3 n}{2 t}=-\langle\sigma v\rangle n^{2}, \quad t_{f}<t<t_{e q}$

$\frac{d n}{d t}+\frac{3 n}{a} \frac{d a}{d t}=-\langle\sigma v\rangle n^{2}, \quad t>t_{e q}$,

with the initial condition $n\left(t_{f}\right)=n_{0}\left(T_{f}\right)$. Here $a=a(t)$ is the scale factor in the Robertson-Walker line element. One might use the following set of equations for $t>$ $t_{e q}$

$\frac{d n}{d t}+\frac{2 n}{t}=-\langle\sigma v\rangle n^{2}, \quad t_{e q}<t<t_{\Lambda}$, 
$\frac{d n}{d t}+\frac{2 n}{\tau_{\Lambda}} \operatorname{coth}\left(\frac{t}{\tau_{\Lambda}}\right)=-\langle\sigma v\rangle n^{2}, \quad t>t_{\Lambda}$,

with the time constant (for $\Lambda=0.76 \varrho_{c}=$ $4.27 \mathrm{keV} / \mathrm{cm}^{3}$ )

$\tau_{\Lambda}=2 m_{\text {Planck }} \sqrt{\frac{c}{3 \hbar \Lambda}}=3.23 \times 10^{17} \mathrm{~s}$,

and $t_{\Lambda}$ following from

$$
\begin{aligned}
1+z_{\Lambda} & \equiv \frac{a\left(t_{0}\right)}{a\left(t_{\Lambda}\right)}=\left(\frac{\sinh \left(t_{0} / \tau_{\Lambda}\right)}{\sinh \left(t_{\Lambda} / \tau_{\Lambda}\right)}\right)^{2 / 3} \\
& =\left(\frac{\Omega_{\Lambda}}{\Omega_{m}}\right)^{1 / 3}=1.47
\end{aligned}
$$

see e.g. the appendix to reference [57]. However, we will see from the solution of equation (33) that $n\left(t_{e q}\right)\langle\sigma v\rangle t_{e q}<10^{-9} \ll 1$, see equation (39) below. Therefore both $n(t)\langle\sigma v\rangle t \ll 1$ and $n(t)\langle\sigma v\rangle \tau_{\Lambda} \tanh \left(t / \tau_{\Lambda}\right) \ll$ 1 for $t \geq t_{e q}$, i.e. the expansion term dominates strongly over the annihilation term for $t>t_{e q}$. This yields standard cold dark matter evolution for late times,

$n(t)=n\left(t_{e q}\right)\left(\frac{a\left(t_{e q}\right)}{a(t)}\right)^{3}, \quad t>t_{e q}$.

We define $\xi=m_{S} c^{2} / k_{B} T_{f}$. The LeeWeinberg condition (32) takes the following form

$$
\begin{aligned}
\exp (\xi)= & \frac{2 b k_{B}^{2} m_{S} c^{2}}{(\sqrt{2 \pi} \hbar c)^{3}}\langle\sigma v\rangle \frac{\sqrt{\xi}}{\xi-1.5} \\
= & 4.18 \times 10^{11} \frac{\langle\sigma v\rangle}{10^{-24} \mathrm{~cm}^{3} / \mathrm{s}} \\
& \times \frac{m_{S} c^{2}}{100 \mathrm{GeV}} \frac{\sqrt{\xi}}{\xi-1.5} .
\end{aligned}
$$

We are interested in a perturbative Higgs portal $\eta^{2} \lesssim 0.1$ and weak scale singlet masses. Our previous results on cross sections then imply that the factor $\left(\langle\sigma v\rangle / 10^{-24} \mathrm{~cm}^{3} \mathrm{~s}^{-1}\right) \times\left(m_{S} / 100 \mathrm{GeV}\right)$ should be in the range between 0.1 and 10 .
This will yield values for $\xi$ between 20 and 30. Thermal theories of particle creation using equation (27) generically predict that particles will remain thermal until the temperature has dropped to a value well below their mass threshold.

Integration of equation (33) yields

$$
\begin{aligned}
n\left(t_{e q}\right)= & {\left[\frac{1}{n\left(t_{f}\right)}\left(\frac{t_{e q}}{t_{f}}\right)^{3 / 2}+2\langle\sigma v\rangle t_{f}\right.} \\
& \left.\times\left(\left(\frac{t_{e q}}{t_{f}}\right)^{3 / 2}-\frac{t_{e q}}{t_{f}}\right)\right]^{-1} \\
\simeq & \frac{n\left(t_{f}\right)}{1+2 n\left(t_{f}\right)\langle\sigma v\rangle t_{f}}\left(\frac{t_{f}}{t_{e q}}\right)^{3 / 2},
\end{aligned}
$$

where we used that freeze-out temperatures following from (35) will at least be a few $\mathrm{GeV}$ for the parameter range of interest here, and therefore $\left(t_{e q} / t_{f}\right)^{1 / 2}>10^{9}$.

The relation between temperature and time and the definition of $\xi$ imply

$$
\begin{aligned}
t_{f} & =\frac{b}{T_{f}^{2}}=\frac{b k_{B}^{2}}{m_{S}^{2} c^{4}} \xi^{2} \\
& =2.53 \times 10^{-11} \xi^{2} \mathrm{~s} \times\left(\frac{100 \mathrm{GeV}}{m_{S} c^{2}}\right)^{2} .
\end{aligned}
$$

This yields

$$
\begin{aligned}
\langle\sigma v\rangle t_{f}= & 2.53 \times 10^{-35} \xi^{2} \mathrm{~cm}^{3} \\
& \times\left(\frac{100 \mathrm{GeV}}{m_{S} c^{2}}\right)^{2} \times \frac{\langle\sigma v\rangle}{10^{-24} \mathrm{~cm}^{3} / \mathrm{s}} .
\end{aligned}
$$

On the other hand, the density is with (35)

$$
\begin{aligned}
n\left(t_{f}\right)= & \left(\frac{m_{S} c}{\sqrt{2 \pi \xi \hbar}}\right)^{3} \exp (-\xi) \\
= & \frac{m_{S}^{2} c^{4}}{2 b k_{B}^{2}\langle\sigma v\rangle} \frac{\xi-1.5}{\xi^{2}} \\
= & 1.98 \times 10^{34} \frac{\xi-1.5}{\xi^{2}} \mathrm{~cm}^{-3} \\
& \times\left(\frac{m_{S} c^{2}}{100 \mathrm{GeV}}\right)^{2} \times \frac{10^{-24} \mathrm{~cm}^{3} / \mathrm{s}}{\langle\sigma v\rangle},
\end{aligned}
$$


and therefore

$2 n\left(t_{f}\right)\langle\sigma v\rangle t_{f}=\xi-1.5$.

This equation also implies

$$
\begin{aligned}
n\left(t_{e q}\right)\langle\sigma v\rangle t_{e q} & =\frac{n\left(t_{f}\right)\langle\sigma v\rangle t_{f}}{1+2 n\left(t_{f}\right)\langle\sigma v\rangle t_{f}} \sqrt{\frac{t_{f}}{t_{e q}}} \\
& =\frac{\xi-1.5}{2 \xi-1} \sqrt{\frac{t_{f}}{t_{e q}}}<10^{-9}
\end{aligned}
$$

and therefore the annihilation term is much smaller than the expansion term for $t \geq t_{e q}$, $d\left(n a^{3}\right) / d t=0$,

$n\left(t_{0}\right)=n\left(t_{e q}\right) z_{e q}^{-3}$,

where

$z_{e q} \equiv \frac{a\left(t_{0}\right)}{a\left(t_{e q}\right)}-1 \simeq \frac{a\left(t_{0}\right)}{a\left(t_{e q}\right)}$.

We use $z_{e q}=3000$ for numerical work.

Equations (36), (37), (38), and (40) yield the current energy density in one singlet species,

$$
\begin{aligned}
\varrho_{S}^{(1)} & =n\left(t_{e q}\right) m_{S} c^{2} \\
& =\frac{2 \xi-3}{2 \xi-1} \xi \frac{k_{B} \sqrt{b}}{2\langle\sigma v\rangle t_{e q}^{3 / 2} z_{e q}^{3}} \\
& \simeq \frac{2 \xi-3}{2 \xi-1} \xi \times \frac{2.51 \mathrm{eV} / \mathrm{cm}^{3}}{\langle\sigma v\rangle / 10^{-24} \mathrm{~cm}^{3} / \mathrm{s}} .
\end{aligned}
$$

However, we can have an $O(N)$ symmetric $N$-plet of electroweak singlets of mass $m_{S}$, such that the current energy density in singlets is

$\varrho_{S} \simeq \frac{2 \xi-3}{2 \xi-1} \xi \times \frac{N \times 2.51 \mathrm{eV} / \mathrm{cm}^{3}}{\langle\sigma v\rangle / 10^{-24} \mathrm{~cm}^{3} / \mathrm{s}}$.

The condition $\varrho_{S}=\varrho_{d m}=1.106 \mathrm{keV} / \mathrm{cm}^{3}$ then determines $\xi$ in terms of $\langle\sigma v\rangle / N$, and substitution in equation (35) then relates $m_{S}, m_{h}$, and $N$. We report the resulting singlet masses in the mass range

\begin{tabular}{|l|l|l|} 
& $m_{h}=115 \mathrm{GeV}$ & $m_{h}=160 \mathrm{GeV}$ \\
\hline$N=1$ & $m_{S}=879 \mathrm{GeV}$ & $m_{S}=883 \mathrm{GeV}$ \\
& $T_{f}=35.9 \mathrm{GeV}$ & $T_{f}=36.1 \mathrm{GeV}$ \\
& $x_{\sigma}=0.0531$ & $x_{\sigma}=0.0531$ \\
\hline$N=10$ & $m_{S}=273 \mathrm{GeV}$ & $m_{S}=285 \mathrm{GeV}$ \\
& $T_{f}=10.7 \mathrm{GeV}$ & $T_{f}=11.1 \mathrm{GeV}$ \\
& $x_{\sigma}=0.557$ & $x_{\sigma}=0.558$ \\
\hline
\end{tabular}

Table 1: Singlet masses in the mass range $m_{W}<m_{S}<1 \mathrm{TeV}$ which satisfy the LeeWeinberg condition (35) for $\eta^{2}=0.1, m_{h}=$ $115 \mathrm{GeV}$ or $m_{h}=160 \mathrm{GeV}$, and $N=1$ or $N=10$.

\begin{tabular}{|l|l|l|} 
& $m_{h}=115 \mathrm{GeV}$ & $m_{h}=160 \mathrm{GeV}$ \\
\hline$N=1$ & $m_{S}=293 \mathrm{GeV}$ & $m_{S}=304 \mathrm{GeV}$ \\
& $T_{f}=12.6 \mathrm{GeV}$ & $T_{f}=13.0 \mathrm{GeV}$ \\
& $x_{\sigma}=0.0505$ & $x_{\sigma}=0.0506$ \\
\hline$N=10$ & $\mathrm{n} / \mathrm{a}$ & $m_{S}=111 \mathrm{GeV}$ \\
& & $T_{f}=4.47 \mathrm{GeV}$ \\
& & $x_{\sigma}=0.540$ \\
\hline
\end{tabular}

Table 2: Singlet masses in the mass range $m_{W}<m_{S}<1 \mathrm{TeV}$ which satisfy the LeeWeinberg condition (35) for $\eta^{2}=0.01, m_{h}=$ $115 \mathrm{GeV}$ or $m_{h}=160 \mathrm{GeV}$, and $N=1$ or $N=10$.

$m_{W}<m_{S}<1 \mathrm{TeV}$ for $\eta^{2}=0.1$ and for $N=1$ or $N=10$ in table 1 . We also report the corresponding freeze out temperatures. The annihilation cross sections are given in the form

$x_{\sigma} \equiv \frac{v \sigma_{S S}}{10^{-24} \mathrm{~cm}^{3} / \mathrm{s}}$.

Table 2 shows solutions in the mass range $m_{W}<m_{S}<1 \mathrm{TeV}$ for very weak coupling $\eta^{2}=0.01$.

The cross sections in table 2 for given $m_{h}$ and $N$ are similar to the cross sections in table 1 , in spite of the weaker coupling. 
This is due to the fact that the corresponding singlet masses in table 2 are smaller and much closer to the peaks in the cross section.

For the flux calculations for $N>1$, we have to rescale the flux (9) by a factor $1 / N$, because the density factor $n^{2}$ for each annihilating species is now suppressed $\propto N^{-2}$, but there are $N$ annihilating species of singlets of mass $m_{S}$. Therefore we find with equations (9) and (15)

$$
\begin{aligned}
j_{\gamma}= & \frac{1.95 \times 10^{20}}{N} \times\left(\frac{\mathrm{GeV}}{m_{S}}\right)^{2} \\
& \times \frac{d \mathcal{N}_{\gamma}\left(E_{\gamma}\right)}{d E_{\gamma}} \frac{v \sigma_{S S}}{\mathrm{~cm}^{5} \mathrm{sr}} \\
= & \frac{8.19 \times 10^{19}}{N} \times\left(\frac{\mathrm{GeV}}{m_{S}}\right)^{2} \\
& \times \frac{\sqrt{m_{S}} \exp \left(-8 E_{\gamma} / m_{S}\right)}{E_{\gamma}^{1.5}+0.00014 m_{S}{ }^{1.5}} \frac{v \sigma_{S S}}{\mathrm{~cm}^{5} \mathrm{sr}} .
\end{aligned}
$$

The parameter $\left(v \sigma_{S S} / N\right) /\left(10^{-24} \mathrm{~cm}^{3} / \mathrm{s}\right)=$ $x_{\sigma} / N$ varies only in the range $5.05 \times 10^{-2} \leq$ $x_{\sigma} / N \leq 5.58 \times 10^{-2}$ for the solutions in tables 1 and 2 . However, $j_{\gamma}$ scales with $m_{S}{ }^{-1.5}$, and therefore the low-mass solutions from table 2 yield higher flux than the solutions from table 1 . We will give results for $j_{\gamma}$ in the case $\eta^{2}=0.1, N=10$, $m_{h}=160 \mathrm{GeV}$ and $m_{S}=285 \mathrm{GeV}$, and also in the case $\eta^{2}=0.01, N=10$, $m_{h}=160 \mathrm{GeV}$ and $m_{S}=111 \mathrm{GeV}$.

The expected contribution to the photon flux below $120 \mathrm{GeV}$ in units of the EGRET flux is shown in figures 8 and 9 for the two cases. The corresponding number of photons per $\mathrm{GeV} \cdot \mathrm{cm}^{2} \cdot \mathrm{s} \cdot \mathrm{sr}$ is given in figures 10 and 11 , respectivley.

The integrated photon flux

$\Phi_{\gamma}\left(E_{\gamma}\right)=\int_{E_{\gamma}}^{\infty} d E j_{\gamma}(E)$

in the two cases is shown in figures 12 and

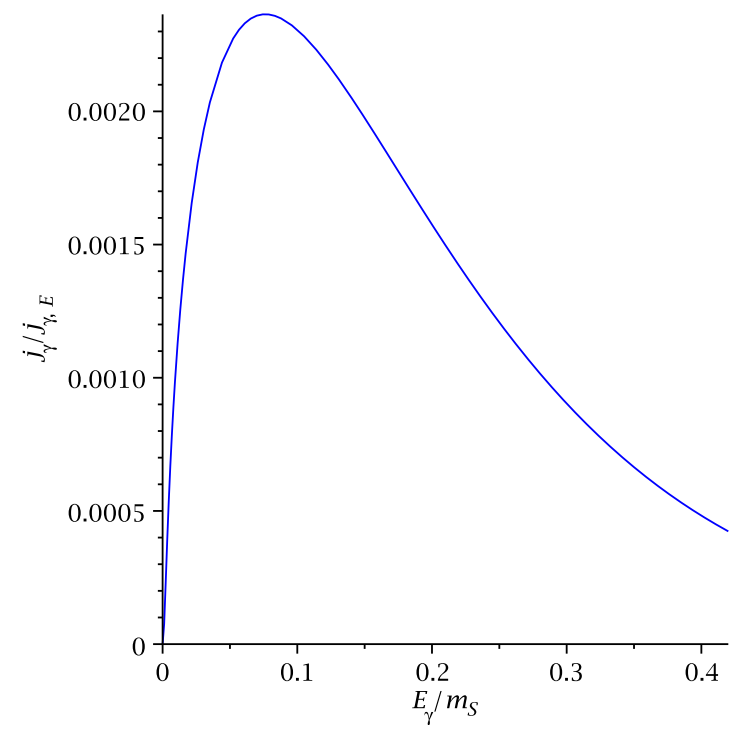

Fig. 8. The photon flux $j_{\gamma}$ in the continuous spectrum for $\eta^{2}=0.1, N=10$, $m_{h}=160 \mathrm{GeV}, m_{S}=285 \mathrm{GeV}$ and $E_{\gamma}<120 \mathrm{GeV}$ in units of the EGRET flux.

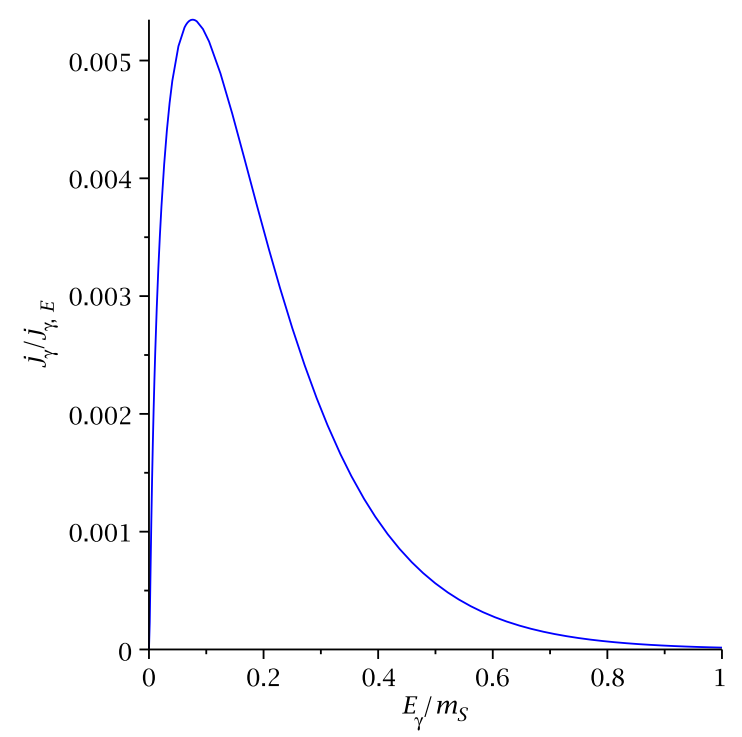

Fig. 9. The photon flux $j_{\gamma}$ in the continuous spectrum for $\eta^{2}=0.01, N=10$, $m_{h}=160 \mathrm{GeV}, m_{S}=111 \mathrm{GeV}$ and $E_{\gamma}<111 \mathrm{GeV}$ in units of the EGRET flux.

13.

For an instrument with an effective area of $8000 \mathrm{~cm}^{2}$ and a field of view of $2.4 \mathrm{sr}$, comparable to the Large Area Telescope on GLAST, the photon flux $j_{\gamma}$ in figure 


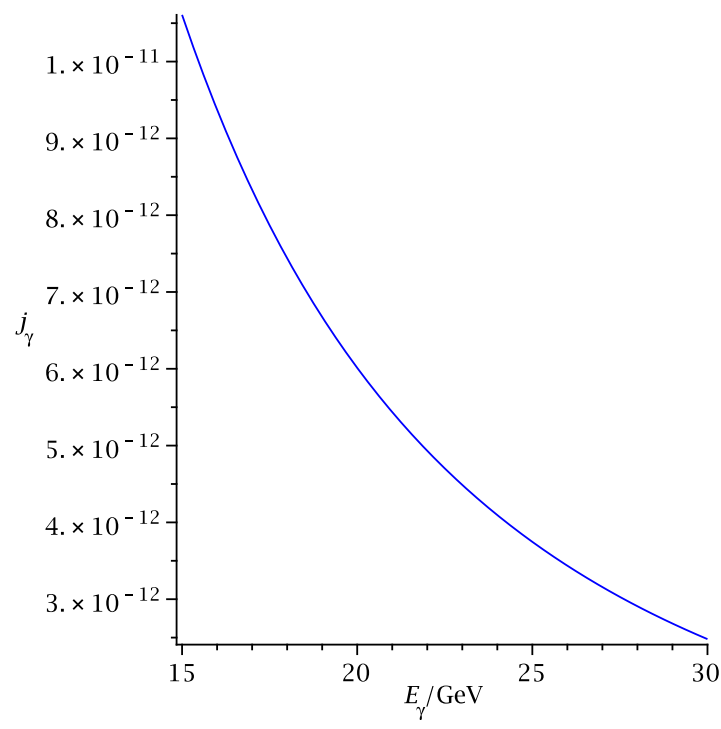

Fig. 10. The photon flux $j_{\gamma}$ in units of $\left(\mathrm{GeV} \mathrm{cm}^{2} \mathrm{ssr}\right)^{-1}$ for $\eta^{2}=0.1, N=10$, $m_{h}=160 \mathrm{GeV}, m_{S}=285 \mathrm{GeV}$ and $E_{\gamma}$ between $15 \mathrm{GeV}$ and $30 \mathrm{GeV}$. The maximum in figure 8 corresponds to $E_{\gamma} \simeq 22 \mathrm{GeV}$.

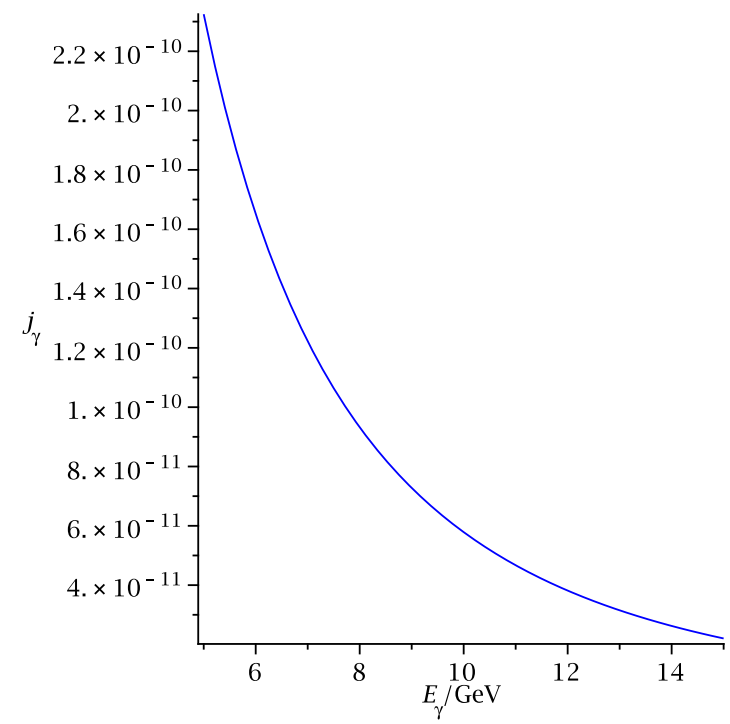

Fig. 11. The photon flux $j_{\gamma}$ in units of $\left(\mathrm{GeV} \mathrm{cm}^{2} \mathrm{ssr}\right)^{-1}$ for $\eta^{2}=0.01, N=10$, $m_{h}=160 \mathrm{GeV}, m_{S}=111 \mathrm{GeV}$ and $E_{\gamma}$ between $5 \mathrm{GeV}$ and $15 \mathrm{GeV}$. The maximum in figure 9 corresponds to $E_{\gamma} \simeq 9 \mathrm{GeV}$.

11 corresponds to an annual rate of 470 photons with energies between $5 \mathrm{GeV}<$ $E_{\gamma}<15 \mathrm{GeV}$ from dark matter annihila-

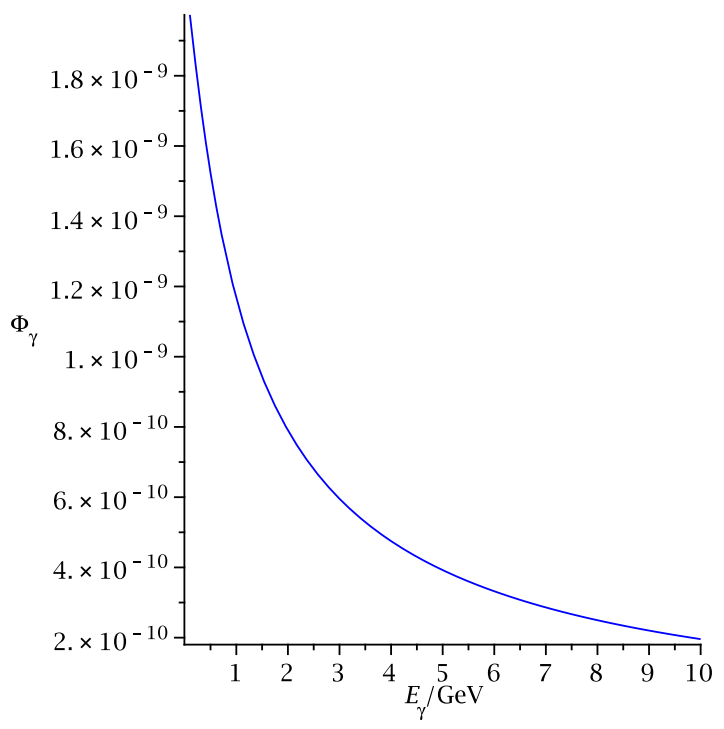

Fig. 12. The integrated photon flux $\Phi_{\gamma}$ above energy $E_{\gamma}$ in units of $\left(\mathrm{cm}^{2} \mathrm{ssr}\right)^{-1}$ for $\eta^{2}=0.1$, $N=10, m_{h}=160 \mathrm{GeV}, m_{S}=285 \mathrm{GeV}$ and $E_{\gamma}$ between $100 \mathrm{MeV}$ and $10 \mathrm{GeV}$.



Fig. 13. The integrated photon flux $\Phi_{\gamma}$ above energy $E_{\gamma}$ in units of $\left(\mathrm{cm}^{2} \mathrm{ssr}\right)^{-1}$ for $\eta^{2}=0.01, N=10, m_{h}=160 \mathrm{GeV}$, $m_{S}=111 \mathrm{GeV}$ and $E_{\gamma}$ between $100 \mathrm{MeV}$ and $10 \mathrm{GeV}$.

tion on a diffuse astrophysical background of 90,000 photons per year. The flux in figure 10 corresponds to an annual rate of 48 photons with energies between $15 \mathrm{GeV}<$ 
$E_{\gamma}<30 \mathrm{GeV}$ from dark matter annihilation on a diffuse astrophysical background of 22500 photons per year. There are two characteristic features which would help to identify $j_{\gamma}$ as an excess due to dark matter annihilation. The excess would extend over an energy range of order $10 \mathrm{GeV}$, and it would be correlated with the galactic halo.

\section{Conclusions}

We have considered perturbatively coupled electroweak singlet dark matter in the intermediate mass range $m_{W}<m_{S}<$ $1 \mathrm{TeV}$. The leading order annihilation cross section of the singlets is enhanced and varies strongly due to proximity to the $W, Z$ and Higgs peaks. The product $v \sigma_{S S}$ is of order $\eta^{2} \times 10^{-23} \mathrm{~cm}^{3} / \mathrm{s}$ for $m_{S}$ close to $m_{h}$, i.e. it can substantially exceed standard estimates of dark matter annihilation cross sections even for perturbative singlet-Higgs coupling.

For singlet masses above the $S S \rightarrow W W$ threshold and electroweak strength coupling $\eta^{2} \simeq 0.1$, the Lee-Weinberg condition and the requirement $\Omega_{S}=\Omega_{d m}$ push $m_{S}$ to high mass values around $900 \mathrm{GeV}$ if there is only one singlet state. However, if there is an $N$-plet of electroweak singlets or if the coupling is weaker, $\eta^{2} \lesssim 0.01$, lower singlet mass values can be achieved, and the annihilation signal from the continuous $\gamma$ ray spectrum can reach a level of several per mil of the EGRET diffuse $\gamma$ ray signal for photon energies $E_{\gamma} \sim 0.08 m_{S}$. This excess contribution over the expected cosmological background would appear typically over an energy range of order 10 $\mathrm{GeV}$, and its dark matter signature would be its correlation with the galactic halo.

The flux reported by EGRET was the diffuse $\gamma$ ray flux after subtraction of then known or expected galactic components.
Reduction of the diffuse "excess" $\gamma$ ray flux due to subtraction of a larger component from interstellar gas and standard extragalactic sources increases the relative importance of an annihilation signal for any possible excess signal and improves detectability. The diffuse $\gamma$ ray flux will be measured with higher sensitivity and precision in the near future by the Large Area Telescope aboard the GLAST satellite. This will also cover a larger energy range up to $300 \mathrm{GeV}$.

The minimal dark matter models considered here include four basic parameters, the singlet mass $m_{S}$, the number of singlet states $N$, the singlet-Higgs coupling $\eta$, and the Higgs mass $m_{h}$. The assumption that electroweak singlets of mass $m_{S}$ provide the dark matter in the universe relates these parameters through Lee-Weinberg theory. Measuring the Higgs mass at the Tevatron or the LHC will reduce the number of free parameters in this class of minimal dark matter models to two, or maybe even to only one free parameter if a missing energy signal can be used to constrain a combination of $\eta, N$ and $m_{S}$. The anticipated smallness of singlet annihilation signals in cosmic $\gamma$ rays indicates that observation of the Higgs particle at the Tevatron or the LHC may be needed for a successful search for a singlet annihilation signal in cosmic rays.

\section{Acknowledgement}

This work was supported by NSERC Canada. RD thanks Freddy Cachazo, Rob Myers and Tom Waterhouse for interesting discussions, and gratefully acknowledges the hospitality of the Perimeter Institute for Theoretical Physics. We also thank the referee for constructive criticism of a first draft of this manuscript. 


\section{References}

[1] J. Preskill, M.B. Wise, F. Wilczek, Phys. Lett. B 120 (1983) 127; L.F. Abbott, P. Sikivie, Phys. Lett. B 120 (1983) 133; M. Dine, W. Fischler, Phys. Lett. B 120 (1983) 137.

[2] S. Weinberg, Phys. Rev. Lett. 50 (1983) 387; H. Goldberg, Phys. Rev. Lett. 50 (1983) 1419; J.R. Ellis, J.S. Hagelin, D.V. Nanopoulos, K.A. Olive, M. Srednicki, Nucl. Phys. B 238 (1984) 453.

[3] E.W. Kolb, R. Slansky, Phys. Lett. B 135 (1984) 378.

[4] Y.M. Cho, Phys. Rev. D 41 (1990) 2462; R. Dick, Mod. Phys. Lett. A 12 (1997) 47; Fortschr. Phys. 45 (1997) 537; Y.M. Cho, Y.Y. Keum, Mod. Phys. Lett. A 13 (1998) 109; Class. Quantum Grav. 15 (1998) 907; Y.M. Cho, J.H. Kim, Dilaton as a dark matter candidate and its detection, arXiv:0711.2858 [gr-qc].

[5] D.J. Chung, E.W. Kolb, A. Riotto, Phys. Rev. Lett. 81 (1998) 4048; Phys. Rev. D 59 (1999) 023501.

[6] V.A. Kuzmin, I.I. Tkachev, Phys. Rev. D 59 (1999) 123006; Phys. Rep. 320 (1999) 199.

[7] L. Kofman, A.D. Linde, A.A. Starobinsky, Phys. Rev. Lett. 73 (1994) 3195; Phys. Rev. D 56 (1997) 3258.

[8] G. Bertone, D. Hooper, J. Silk, Phys. Rep. 405 (2005) 279.

[9] D. Hooper, T. Plehn, Phys. Lett. B 562 (2003) 18.

[10] A. Bottino, F. Donato, N. Fornengo, S. Scopel, Phys. Rev. D 68 (2003) 043506.

[11] V. Silveira, A. Zee, Phys. Lett. B 161 (1985) 136.

[12] J. McDonald, Phys. Rev. D 50 (1994) 3637.
[13] C.P. Burgess, M. Pospelov, T. ter Veldhuis, Nucl. Phys. B 619 (2001) 709.

[14] H. Davoudiasl, R. Kitano, T. Li, H. Murayama, Phys. Lett. B 609 (2005) 117.

[15] B. Patt, F. Wilczek, Higgs-field portal into hidden sectors, hep-ph/0605188.

[16] D.E. Holz, A. Zee, Phys. Lett. B 517 (2001) 239.

[17] C. Bird, R. Kowalewski, M. Pospelov, Mod. Phys. Lett. A 21 (2006) 457.

[18] H. Murayama, Physics beyond the Standard Model and dark matter, arXiv:0704.2276 [hep-ph].

[19] M. Cirelli, N. Fornengo, A. Strumia, Nucl. Phys. B 753 (2006) 178; M. Cirelli, A. Strumia, M. Tamburini, Nucl. Phys. B 787 (2007) 152.

[20] M. Cirelli, R. Franceschini, A. Strumia, Nucl. Phys. B 800 (2008) 204.

[21] J. McDonald, N. Sahu, JCAP 0806 (2008) 026 .

[22] O. Bertolami, R. Rosenfeld, The Higgs portal and a unified model for dark energy and dark matter, arXiv:0708.1784 [hep$\mathrm{ph}$.

[23] W.F. Chang, J.N. Ng, J.M.S. Wu, Phys. Rev. D 74 (2006) 095005; Phys. Rev. D 75 (2007) 115016; Phenomenology from a U(1) gauged hidden sector, arXiv:0706.2345 [hep-ph].

[24] K.A. Meissner, H. Nicolai, Phys. Lett. B 648 (2007) 312.

[25] J.R. Espinosa, M. Quirós, Phys. Rev. D 76 (2007) 076004.

[26] S. Profumo, M.J. Ramsey-Musolf, G. Shaughnessy, JHEP 08 (2007) 010.

[27] A. Datta, A. Raychaudhuri, Phys. Rev. D 57 (1998) 2940. 
[28] H. Davoudiasl, T. Han, H.E. Logan, Phys. Rev. D 71 (2005) 115007

[29] R. Schabinger, J.D. Wells, Phys. Rev. D 72 (2005) 093007.

[30] John March-Russell, Stephen M. West, Daniel Cumberbatch, Dan Hooper, Heavy dark matter through the Higgs portal, arXiv:0801.3440 [hep-ph].

[31] P. Blasi, R. Dick, E.W. Kolb, Astropart. Phys. 18 (2002) 57.

[32] P. Ullio, L. Bergström, J. Edsjö, C. Lacey, Phys. Rev. D 66 (2002) 123502.

[33] L. Bergström, P. Ullio, J.H. Buckley, Astropart. Phys. 9 (1998) 137.

[34] N.W. Evans, F. Ferrer, S. Sarkar, Phys. Rev. D 69 (2004) 123501.

[35] J.F. Navarro, C.S. Frenk, S.D.M. White, Mon. Not. R. Astron. Soc. 275 (1995) 720; Astrophys. J. 462 (1996) 563.

[36] A. Klypin, H. Zhao, R.S. Somerville, Astrophys. J. 573 (2002) 597.

[37] A. Boyarski, A. Neronov, O. Ruchayskiy, M. Shaposhnikov, I. Tkachev, Phys. Rev. Lett. 97 (2006) 261302.

[38] Higher Transcendental Functions Vol. 1, ed. A. Erdélyi (McGraw-Hill, New York 1953) pp. 31-32.

[39] Handbook of Mathematical Functions, eds. M. Abramowitz and I.A. Stegun (United States National Bureau of Standards, 10th printing, Washington 1972) pp. 1004-1005.

[40] O. Biebel, D. Milstead, P. Nason, B.R. Webber, Fragmentation functions in $e^{+} e^{-}$ annihilations and lepton-nucleon DIS, in [53].

[41] D. Bender et al. (HRS Collaboration), Phys. Rev. D 31 (1985) 1.
[42] L. Bergström, J. Edsjö, P. Ullio, Phys. Rev. Lett. 87 (2001) 251301.

[43] M. Kaplinghat, L. Knox, M.S. Turner, Phys. Rev. Lett. 85 (2000) 3335.

[44] J.F. Beacom, N.F. Bell, G.D. Mack, Phys. Rev. Lett. 99 (2007) 231301.

[45] H. Yüksel, S. Horiuchi, J.F. Beacom, S. Ando, Phys. Rev. D 76 (2007) 123506.

[46] S. Weinberg, The Quantum Theory of Fields Vol. 1 (Cambridge University Press, Cambridge 1995) Sec. 3.7.

[47] K. Griest, M. Kamionkowski, Phys. Rev. Lett. 64 (1990) 615; L. Hui, Phys. Rev. Lett. 86 (2001) 3467.

[48] F.A. Aharonian, W. Wittek et al. (HEGRA Collaboration), Astropart. Phys. 17 (2002) 459.

[49] P. Sreekumar et al., Astrophys. J. 494 (1998) 523.

[50] A.W. Strong, I.V. Moskalenko, O. Reimer, Astrophys. J. 613 (2004) 962.

[51] F.W. Stecker, S.D. Hunter, D.A. Kniffen, Astropart. Phys. 29 (2008) 25.

[52] J.F. Donoghue, E. Golowich, B.R. Holstein, Dynamics of the Standard Model (Cambridge University Press, Cambridge 1992) Sec. XV.2.

[53] W.-M. Yao et al. (Particle Data Group), J. Phys. G 33 (2006) 1 and 2007 partial update for the 2008 edition.

[54] M.P. Sanders, Search for Low Mass SM Higgs at the Tevatron, arXiv:0805.1248 [hep-ex]; A. Duperrin, Review of Searches for Higgs Bosons and Beyond the Standard Model Physics at the Tevatron, arXiv:0805.3624 [hep-ex].

[55] B.W. Lee, S. Weinberg, Phys. Rev. Lett. 39 (1977) 165. 
[56] G. Steigman, Ann. Rev. Nucl. Part. Sci. 29 (1979) 313.

[57] M.G. Barnett, R. Dick, K.E. Wunderle, Mon. Not. R. Astron. Soc. 349 (2004) 1500 . 